\title{
X-rays Image reconstruction using Proximal Algorithm and adapted TV Regularization
}

\author{
Aicha Allag, ${ }^{\mathrm{a}, \mathrm{b}}$, Redouane Drai ${ }^{\mathrm{a}}$, Tarek Boutkedjirt ${ }^{\mathrm{b} * *}$ \\ Abdessalam Benammar ${ }^{\mathrm{b}}$, and Wahiba Djerir ${ }^{\mathrm{a} * *}$ \\ ${ }^{a}$ Research Center in Industrial Technologies CRTI. P.O.Box 64, Cheraga 16014, Algers, Algeria \\ ${ }^{b}$ University of Sciences and Technology Houari Boumediene, BP 32, El-Alia, DZ-6111 Algiers (Algeria)
}

\begin{abstract}
Computed tomography (CT) aims to reconstruct an internal distribution of an object based on projection measurements . In the case of a limited number of projections, the reconstruction problem becomes significantly ill-posed. Practically, reconstruction algorithms play a crucial role in overcoming this problem. In the case of missing or incomplete data, and in order to improve the quality of the reconstruction image, the choice of a sparse regularisation by adding $\boldsymbol{l}_{\boldsymbol{l}}$ norm is needed. The reconstruction problem is then based on using proximal operators. We are interested in the Douglas-Rachford method and employ total variation (TV) regularization. An efficient technique based on these concepts is proposed in this study. The primary goal is to achieve highquality reconstructed images in terms of PSNR parameter and relative error. The numerical simulation results demonstrate that the suggested technique minimizes noise and artifacts while preserving structural information. The results are encouraging and indicate the effectiveness of the proposed strategy.

[copyright information to be updated in production process]

Keywords: Reconstruction, regularization, proximal method, X-rays, ill-posed problem;
\end{abstract}

\section{Introduction}

Several strategies have been developed since the discovery of X-rays and their application in radiography. Such us tomographic imaging, which allows for the visualization of the interior structure of objects.

$\mathrm{X}$-rays passing through the object in different directions gives an internal slice of the object of interest. Tomographic imaging is employed in medicine and nondestructive testing of materials. It allows the possibility to observe the structures of various compositions. This is accomplished using the principle of computed tomography (CT) that allows visualization which should be similar to that of reality. This reconstruction technique is based on mathematical methods. Tomographic reconstruction is mathematically classified as an ill-posed problem. Particularly when a low number of projections is considered $[1,2]$. Image reconstruction using classical aproaches unsuitable for this category of problems, as it leads to unstable reconstruction due to the need for a priori information. To overcome this problem, new researches suggest regularization methods that impose constraints on the solution [3].

During the last years, a new type of iterative reconstruction methods such as the compressed sensing (CS) has been developed. These methods have attracted a lot of interest particularly in the case of undersampled CT reconstruction. Compared to the filtered back-projection algorithm (FBP) method, in the case of limited number of projections, algorithms based on CS, efficiently reconstruct images and reduce artefacts [4, 5].

The convex optimization is based on the minimization of the sum of functions $[6,7,8]$. When one function is differentiable, the proximal operator is one of the most important tools for algorithms. For the optimization of inverse problems, recent methods resulting from a convex optimization approach, such as proximal and sparse representations, are applied. The minimization concerns a sum of convex functions with linear operators and the variables are of large dimensions. The so called first splitting proximal order algorithms find their applications in this field [6]. These splitting methods have found a lot of applications in image processing, such as Forward-backward and DouglasRachfords splitting [8].

The total variation (TV) norm was utilized and introduced in image restoration problems by Rudin, Osher and

\footnotetext{
* Corresponding author. Tel.: +213213420 19; fax: +21321342019.

E-mail address: a.allag@crti.dz.

** Corresponding author. Tel.: +21321342019; fax: +21321342019.

E-mail address: a.allag@crti.dz.
} 
Fatemi [9]. The TV-regularization was introduced in the field of compressed sensing in the work of Candes et al. for exact recovery of an image which has been obtained from sparse samples by solving the $\boldsymbol{l}_{1}$-norm optimization problem [10].

In this paper, we propose a first order technique based on Douglas-Rachford approach associated with TV regularisation and positivity constraint optimization. This method is applied to non-destructive evaluation of materials in the case of a 2D reconstruction of X-rays tomography. The efficiency of the developed algorithm is tested on Shepp Logan and Forbild computerized phantoms, using simulated projections without and with noise. The results are analyzed in terms of the PSNR value.

\section{Theoretical background of $\mathrm{X}$ rays image reconstruction}

Tomographic reconstruction is related to the image of objects from projections which have been obtained by exposing them to X-rays in different orientations. The relationship between the unknown distribution of the object and the measured projections resulting of the Radon transform.

$\mu \in \mathbb{R}^{\mathrm{n}}$ is the unknown distribution, $y \in \mathbb{R}^{\mathrm{m}}$ represent the projections and $A: \mathbb{R}^{\mathrm{n}} \rightarrow \mathbb{R}^{\mathrm{m}}$ is the Radon transform, We can use the following expression:

$$
y=A \mu+w .
$$

The numerical solution of Equation 1 needs discretization,

In the real case, the projections $y$ include an additive noise $w$. Typically, the inverse problem is solved by minimizing a criterion.

In practice, introducing a regularization technique with a priori information is required for reconstructing a noisy image [13]. After that, the solution to equation 1 is found by minimizing the expression :

$$
\min _{\mu \in \mathbb{R}^{n}}\left(\|A \mu-y\|^{2}+\lambda \varphi(\mu)\right) \quad \text { such that } \mu>0
$$

where $\varphi(\mu)$ presents a priori information of the reconstructed image and $\lambda$ is a positive relaxation parameter.

\section{Methods}

\subsection{Douglas-rachford algorithm}

The Douglas-Rachford (DR) method was initially proposed in [11]. The proximal idea was later utilized in the field of signal and image processing [12]. The DR algorithm is an iterative technique that provides for the minimization of the following functional:

$$
\min _{\mu \in \mathbb{R}^{n}}[F(\mu)+G(\mu)]
$$

$\mathrm{F}: \mathbb{R}^{\mathrm{n}} \rightarrow \mathbb{R} \cup\{+\propto\}$ and $\mathrm{G}: \mathbb{R}^{\mathrm{n}} \rightarrow \mathbb{R} \cup\{+\propto\}$ are two convex functions respectively. This approach allows solving the equation (1).

The DR algorithm is gived as follows [3]:

Algorithm 1 (DR):

1: $\tilde{\mu}_{0} \in \mathbb{R}^{\mathrm{n}}, \tau>0, \gamma>0 \quad \tilde{\mathrm{x}}_{0}>0$

2: For each $\mathrm{k}=1,2, \ldots ; \mathrm{N}$

3: $\mu_{\mathrm{k}}=\operatorname{Prox}_{\tau \mathrm{G}}\left(\tilde{\mathrm{x}}_{\mathrm{k}}\right)$

4: $\widetilde{\mathrm{x}}_{\mathrm{k}+1}=\tilde{\mathrm{x}}_{\mathrm{k}}+\gamma\left(\operatorname{Prox}_{\tau \mathrm{F}}\left(2 \mu_{\mathrm{k}}-\tilde{\mathrm{x}}_{\mathrm{k}}\right)-\mu_{\mathrm{k}}\right)$

\subsection{Formulation of the proposed algorithm}

The minimisation in equation (2) can be applied to the sum of several functions F, G, K.etc. In our case, we use the sum of three functions and the minimization is:

$$
\min _{\mu \in \mathbb{R}^{n}}[F(\mu)+G(\mu)+H(\mu)]
$$

In this study, we consider:

$$
\begin{aligned}
& \mathrm{F}(\mu)=\|\mathrm{A} \mu-y\|^{2} \\
& \mathrm{G}(\mu)=\|\mu\|_{1}=\sum_{\mathrm{n}=1}^{\mathrm{N}} \mu_{\mathrm{n}}, \\
& \mathrm{H}(\mu)=\mathrm{J}_{\mathrm{TV}}(\mu)=\sum_{\mathrm{i}, \mathrm{j}}\left\|(\nabla \mu)_{\mathrm{i}, \mathrm{j}}\right\| .
\end{aligned}
$$


Where, $\|\mu\|_{1}$ is the $l_{l}$-norm. The $l_{l}$-norm of the image is the sum of the absolute values of the image pixel values, and its minimization allows sparse solutions $[7,8] . \mathrm{J}_{\mathrm{TV}}(\mu)$ is the discrete representation of the total variation [9]. This function preserves edges and permits reconstructing. TV regularization is mostly used in image reconstruction [14, $15,16,17,26]$ because it allows recovering the edges and contours of the image. For the resolution of equation (2), several methods have been proposed $[18,19,20]$.

In practice, we compute the gradient using the following approximation:

$$
\left|(\nabla \mu)_{i, j}\right| \approx \sqrt{\left(\mu_{i+1, j}-\mu_{i, j}\right)^{2}+\left(\mu_{i, j+1}-\mu_{i, j}\right)^{2}},
$$

where $|$.$| presents the Euclidean norm in \mathrm{R}^{2}$.

The total variation norm of is defined as follows:

$$
\|\mu\|_{T V}=\sum_{i, j} \sqrt{\left(\mu_{i+1, j}-\mu_{i, j}\right)^{2}+\left(\mu_{i, j+1}-\mu_{i, j}\right)^{2}},
$$

and the smoothed TV norm is as follows:

$$
\mathrm{J}_{\mathrm{TV}}^{\mathrm{reg}}(\mu)=\sum_{\mathrm{i}, \mathrm{j}} \sqrt{\left\|(\nabla \mu)_{\mathrm{i}, \mathrm{j}}\right\|^{2}+\varepsilon^{2}},
$$

where $\varepsilon$ is a small positive parameter. The TV regularisation is used in equation (2). For the solution, we need to use an iterative method such as the gradient descent method and the smoothed TV norm. The solution through a gradient descent is expressed as following:

$$
\mu_{k+1}=\mu_{k}+\alpha R^{T}\left(R \mu_{k}-P+\lambda_{k} \operatorname{Grad} \mathrm{J}_{\mathrm{TV}}^{\mathrm{reg}}\left(\mu_{k}\right)\right) .
$$

Where $\alpha$ is a step size.

The gradient of the smoothed TV norm is written as:

$$
\operatorname{Grad} J_{\mathrm{TV}}^{\mathrm{reg}}(\mu)=-\operatorname{div} \mathrm{J}\left(\frac{\nabla \mu}{\sqrt{\varepsilon^{2}+\|\nabla \mu\|^{2}}}\right) .
$$

In order to solve equation (2) in the case of TV minimization, a number of splitting methods have been proposed $[18,21,22,23]$ and the choice of the parameter $\lambda$ in the regularization term was studied and still a challenge [24, 25]. In this paper, we consider the two sequences, $\mu_{\mathrm{k}}$ and $\lambda_{k}$ converging respectively to $\mu_{\mathrm{k}+1}$ and $\lambda_{k+1}$. We propose a selected of parameter $\lambda_{k}$ with an efficient adaptative scheme that computes the sequence:

$$
\lambda_{k+1}=\lambda_{k} /\left(1+2 \lambda_{k}\right) .
$$

The steps of our algorithm so called ADRTV algorithm, include the combination of DR method (algorithm 1) and the gradient descent method with the smoothed TV norm. These steps are detailed in the following subsection.

\subsection{Pseudocode of the ADRTV method}

The pseudocode is presented below. The image variable is denoted $\mu_{\mathrm{k}}$ in each iteration.

1: $\tilde{\mu}_{0} \in \mathbb{R}^{\mathrm{n}}, \tau>0, \gamma>0 \tilde{\mathrm{x}}_{0}>0$

2: For each $\mathrm{k}=1,2, \ldots ; \mathrm{N}$

3: $\mu_{\mathrm{k}}=\operatorname{Prox}_{\mathrm{TG}}\left(\tilde{\mathrm{x}}_{\mathrm{k}}\right)$

4: $\tilde{\mathrm{x}}_{\mathrm{k}+1}=\tilde{\mathrm{x}}_{\mathrm{k}}+\gamma\left(\operatorname{Prox}_{\mathrm{\tau F}}\left(2 \mu_{\mathrm{k}}-\tilde{\mathrm{x}}_{\mathrm{k}}\right)-\mu_{\mathrm{k}}\right)$

5: $\widetilde{\mathrm{x}}_{\mathrm{k}+1}=\widetilde{\mathrm{x}}_{\mathrm{k}}-\nabla \mathrm{F}\left(\tilde{\mathrm{x}}_{\mathrm{k}}+\lambda_{k} \operatorname{Grad~J}\left(\tilde{\mathrm{x}}_{\mathrm{k}}\right)\right)$

6: $\lambda_{k+1}=\lambda_{k} /\left(1+2 \lambda_{k}\right)$

7: $\mu_{\mathrm{k}}=\max \left(\mu_{\mathrm{k}}\right)$

Lines 1-4 solve equation (2) using the DR method. The updated image (line 5) is calculated by using the gradient descent iterative scheme with an adaptively selected parameter $\lambda_{k}$ (line 6). Finally, we propose an improvement of the algorithm by imposing positivity constraint at each iteration (line 7). $\max$ is the value of the positive part of the solution. In the case of constant $\lambda$, the pseudocode tolls to a DRTV algorithm. 


\subsection{Parameter for the evaluation of reconstructed image quality}

The quality of the reconstruction is evaluated by comparing the original image to the reconstructed one. The Peak Signal to Noise Ratio (PSNR) was used as an evaluation criterion. If $\tilde{\mu}$ and $\mu_{0}$ are the reconstructed image and the original image of size $\mathrm{n} \times \mathrm{m}$, respectively, the PSNR in $\mathrm{dB}$ is expressed as follows:

$$
P S N R=10 \log _{10}\left[\frac{D^{2}}{M S E}\right], \text { with } M S E=\frac{\sum_{m, n}^{M, N}\left(\mu_{\tilde{m}, n}-\mu_{m, n}^{0}\right)^{2}}{M N},
$$

And $\boldsymbol{D}$ the maximum possible pixel value of the image. The mean squared error of these two images is denoted by MSE. In order to compare the reconstruction results, the relative error can also be used. Then latter is defined by:

$$
\operatorname{Err}=\left(\frac{\left\|\widetilde{\mu}-\mu_{0}\right\|}{\left\|\mu_{0}\right\|}\right) \text {. }
$$

\section{Simulation and results}

\subsection{Implementation}

The proposed algorithms (DR and DRTV) were initially implemented on synthetic images of a noise-free SheppLogan phantom and a noise-free Forbild phantom (Fig. 1 (a), (b)). The image reconstruction allows us to evaluate the algorithms' resilience according to the number of projections $(\mathrm{P})$. We used a parallel beam imaging geometry to obtain these projections data.
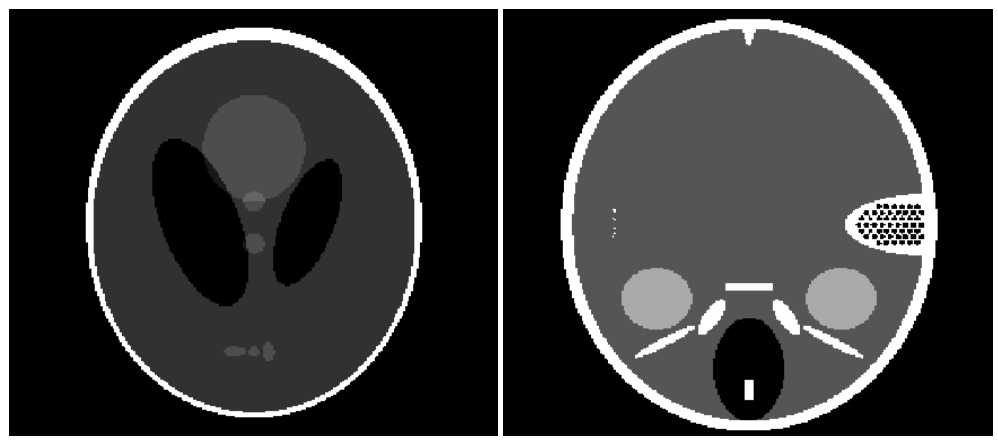

Fig. 1. Shepp-Logan phantom (a) and FORBILD phantom (b)

The reconstruction is obtained using several numbers of projections which are distributed at equal angle with 360 $\circ$. After several tests of the parameters values of the algorithm, the one that provides the highest performance in terms of PSNR have been chosen.

\subsection{Simulation Results}

- $\quad$ Noise-free projection data

Figure 2 (a), (b), (c), and (d) show respectively, the original Shepp Logan synthetic image, the reconstructed images using DR, DRTV, and ADRTV techniques after 2000 iterations, using noise-free projection data. Figure 2 (b) gives a low-quality DR reconstructed image with significant artifacts caused by insufficiency of projection data.

The artifacts in Fig. 2 (c) have been significantly minimized by employing a DRTV technique with fixed to 0.001. The three oval forms at the bottom of the image have blurred edges and some artifacts can be seen in the background region. We can see that the ADRTV method reconstructed a high-quality image compared to the result obtained by DR and DRTV methods. The application of positivity constraint and a TV regularization is efficient in reducing the artifacts of the reconstruction image. The ARTV method can significantly suppress the streak artifacts and also preserve the edge structure information of the reconstructed image.

(a)

(b) $\mathrm{PSNR}=27.04$

(c) $\mathrm{PSNR}=44.79$

(d) $\mathrm{PSNR}=54.36$ 

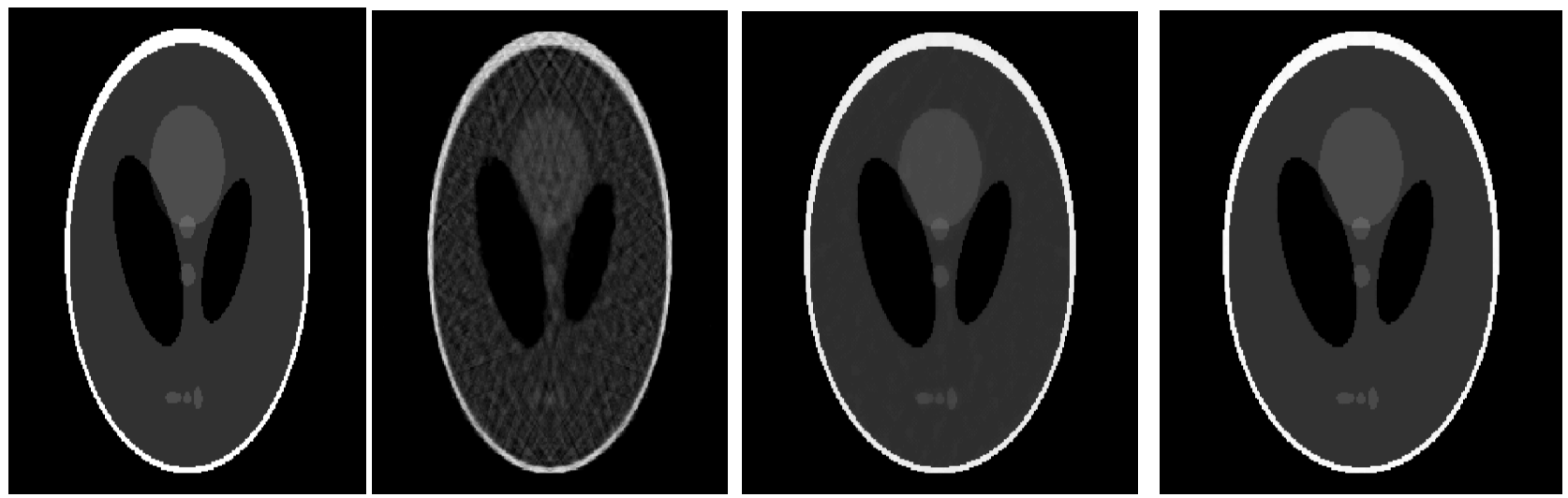

Fig. 2. Reconstructed results of a of Shepp-Logan phantom (a) from 20 projections using DR method (b), DRTV method with fixed $\lambda$ to $0.001(\mathrm{c})$ and ADRTV method (d).

Fig.3 shows zoomed ROI images taken from the Phantom Shepp Logan of Fig. 2. As shown clearly in Fig. 3, the reconstructed image using DR and DRTV methods have more artifacts compared to the reconstructed images obtained by using ADRTV.

Fig. 4 shows the horizontal profiles of line 160 in DRTV and ADRTV reconstructed images corresponding to the Shepp Logan phantom. It can be clearly observed that the ADRTV algorithm gives a closer result to the true image compared to DRTV.

(a)

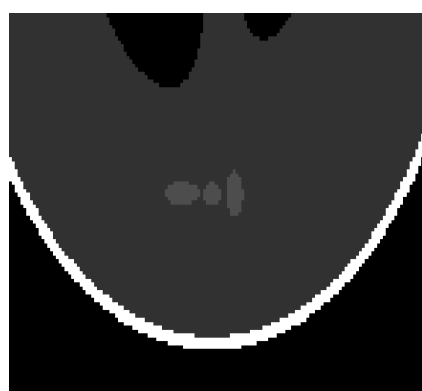

(b)

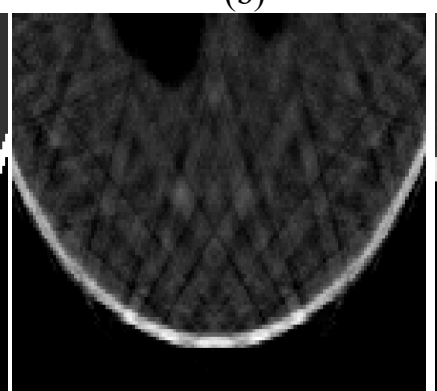

(c)

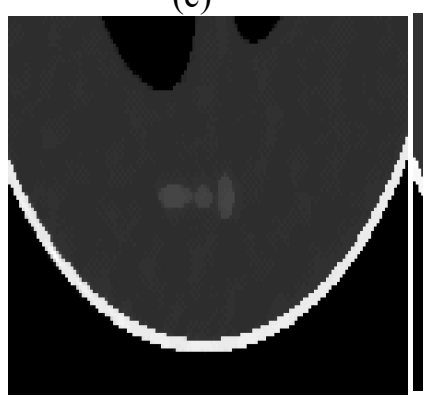

(d)

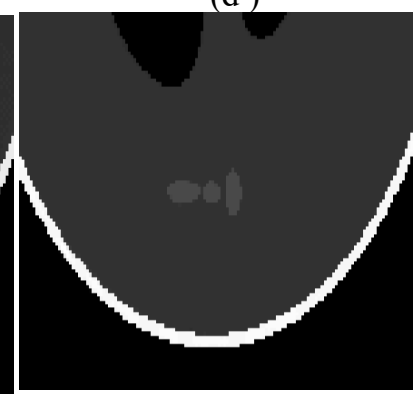

Fig. 3. Zoomed ROI images taken from the Shepp Logan Phantom (a), the reconstructed images using the DR (b), the reconstructed images using the DRTV method with fixed $\lambda=0.001$ (c) and the ADRTV method (d).

(a)

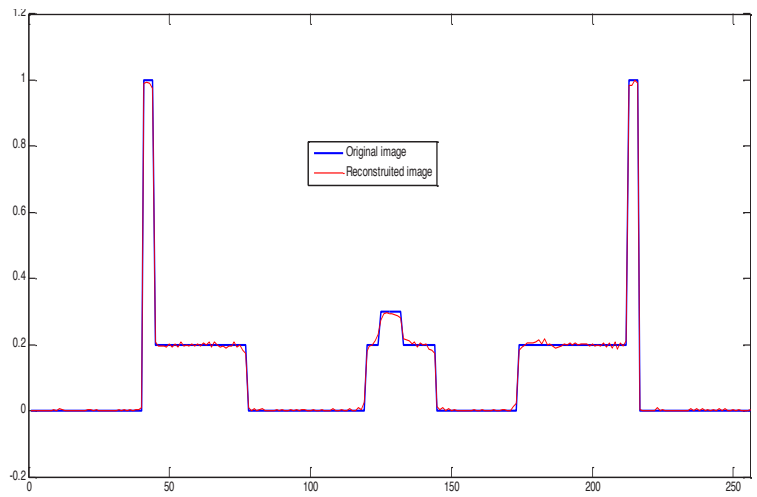

(b)

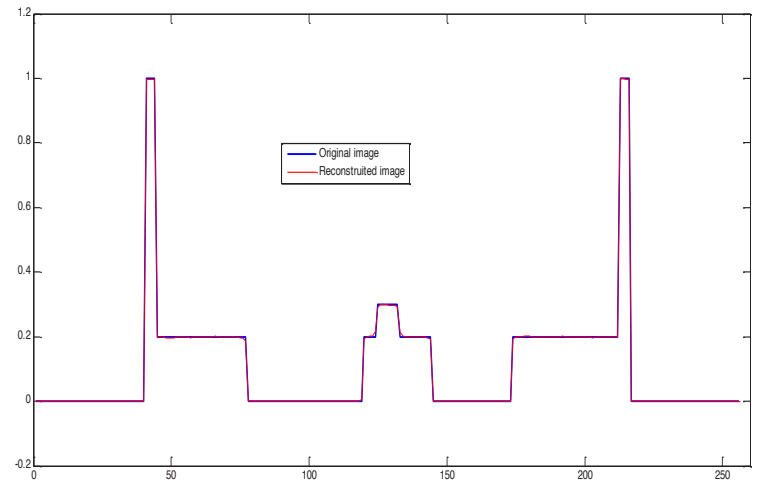

Fig. 4. Horizontal profiles of line 160 in DRTV and ADRTV reconstructed images corresponding to the Shepp 
Logan phantom.

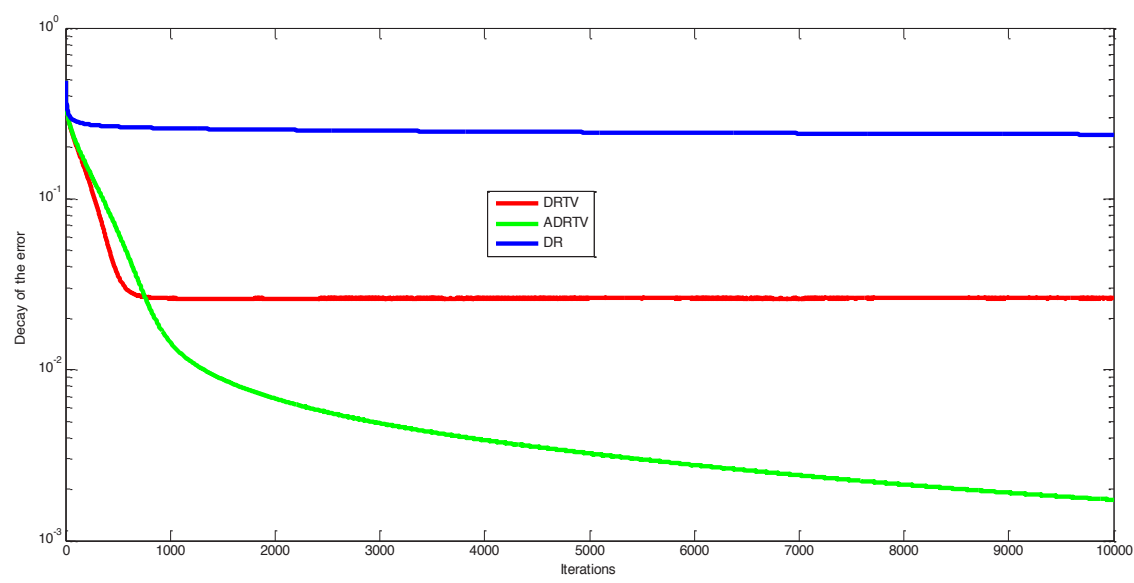

Fig. 5. Relative error $\left(\frac{\left\|\mu_{\mathrm{k}}-\mu_{0}\right\|}{\left\|\mu_{0}\right\|}\right) \mathrm{vs}$ the number of iterations $k$.

To determine the performance of the reconstructed image using DRTV and ADRTV, the PSNR for different projections numbers $(15,20,30,40$ and 60$)$ is calculated. The comparison results are summarized in Table 1.

Table 1. PSNR values of DRTV and ADRTV reconstruction from different numbers of projections in $(\mathrm{dB})$.

\begin{tabular}{lll}
\hline Projections & DRTV & ADRTV \\
\hline 15 & 41.32 & 48.96 \\
20 & 44.79 & 54.36 \\
30 & 68.89 & 70.25 \\
40 & 70.84 & 73.43 \\
60 & 72.79 & 75.27 \\
\hline
\end{tabular}

In the case of low projections number $(\mathrm{P}=15)$ the proposed ADRTV technique leads to a PSNR value of 48.96 against 41.32 DRTV method attains. For greater number of projections, the proposed ADRTV method has achieved PSNR values of $54.36,70.25,73.43$ and 75.27 with $20,30,40$ and 60 projections respectively. The corresponding values for the DRTV are 44.79, 68.89, 70.84 and 72.79. From table 1, it can be noticed that, in comparison with the DRTV method, the ADRTV one enhances the achieved PSNR of $2.46 \mathrm{~dB}$ in the case of 60 projections. This enhancement increases to $7.64 \mathrm{~dB}$ in the case of 15 projections. Hence, the ADRTV image reconstruction method gives better performance results in the image reconstruction than those of DRTV, particularly from low number of projection (15 and 12).

The decreasing of the relative error, $E_{r}\left(\mu_{k}\right)$ for increasing number of iterations for the three methods is presented in Fig. 5 for 20 projections. The reconstructed image with DRTV method was solved with an efficient adaptative scheme of $\lambda$ given in equation (13).

Fig. 5, shows that the ADRTV method can reconstruct high-quality images at less iteration number. The application of this method can thus effectively accelerate the iterative solution. As it can be seen from this figure, the relative error obtained by the DR and the DRTV methods, remain limited to a certain value for above 800 iterations. In contrast, the proposed ADRTV method overcomes such limitation.

In the case of low contrast and critical spatial resolution, we use the above-mentioned tested algorithms on the 2D Forbild phantom [10] in Fig. 6 to demonstrate the performance of the proposed ADRTV approach (a).

(a)

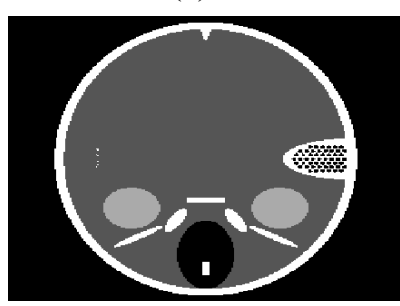

(b) $\mathrm{PSNR}=22.90$

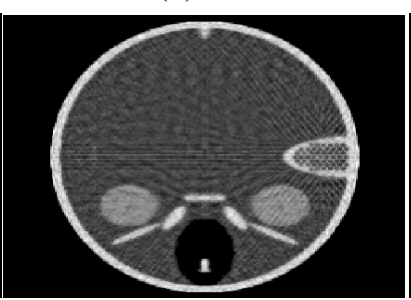

(C) $\mathrm{PSNR}=33.60 \mathrm{db}$

(d) $\mathrm{PSNR}=34.26$
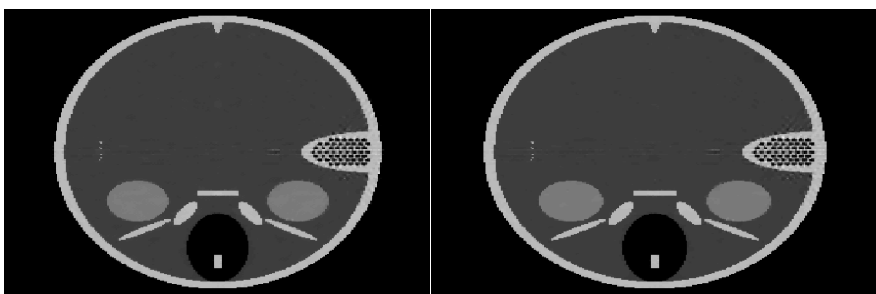
Fig. 6. Reconstructed images of Forbild phantom (a) from 20 projections after 2000 iterations using DR method (b), DRTV method (c) and ADRTV (d).

Fig. 4 (b), (c), and (d) show the reconstructed images, respectively. We can see that the DRTV and ADRTV approaches can provide high-quality images (c) and (d), with the ADRTV approach outperforming the DR one (b).

i. Noisy projection data

In order to test the robustness of the reconstruction methods in a more realistic condition, we have considered a synthetic image from projected data corrupted by a Gaussian noise of a standard deviation $\sigma=0.006$ and $\sigma=0.02$ and a zero mean. By applying the DR algorithm, the reconstruction gave a low quality image with In comparison to the original, there is no visual improvement (Fig. 7). Stripe artefacts can be seen in the reconstructed images. The reconstructed image is distorted by these artefacts. and do not conserve the sharp edges.

$\mathrm{P}=30 \sigma=0.006$

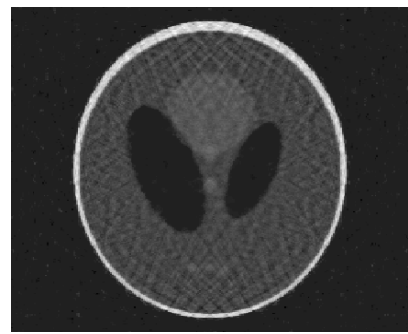

$\mathrm{P}=30 \sigma=0.02$

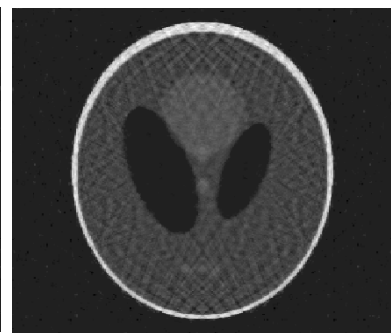

$\mathrm{P}=20 \sigma=0.006$

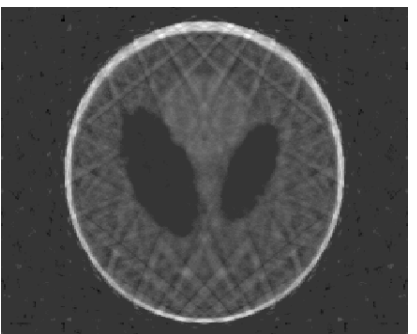

$\mathrm{P}=20 \sigma=0.02$

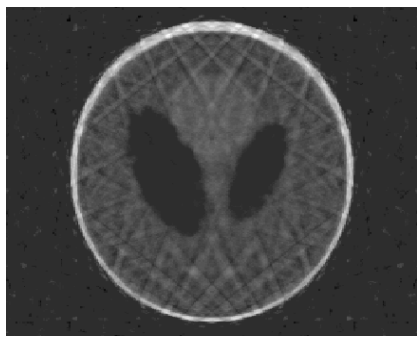

Fig. 7. Shepp-Logan reconstruction image by using DR algorithm from noisy projection data.

$\mathrm{P}=30 \quad \sigma=0.006$

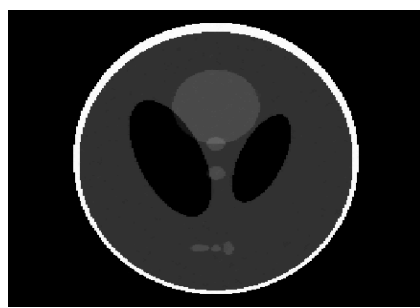

$\mathrm{P}=30 \sigma=0.02$

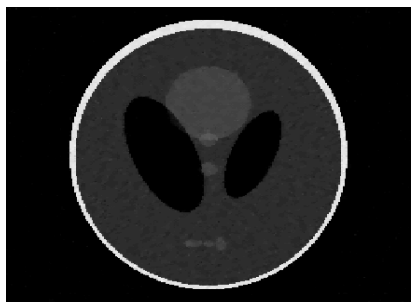

$\mathrm{P}=20 \sigma=0.006$

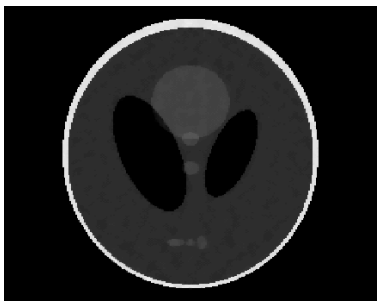

$\mathrm{P}=20 \sigma=0.02$

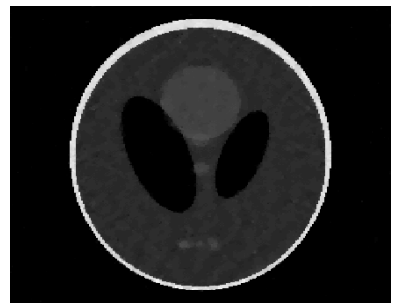

Fig. 8. Shepp-Logan reconstruction image by using ADRTV algorithm from noisy projection data.

In Fig. 8 are presented the images obtained by the application of the ADRTV algorithm. These results show a significant improvement in the image quality (no artifacts).

A comparative study between DRTV and ADRTV algorithms is summarized in Tables 2 for the two values of $\sigma$ respectively where the values of the PSNR were calculated for various projections numbers $(15,20,30,40$ and 60$)$.

Table 2: PSNR values of DRTV and ADRTV reconstruction from noisy projections data $(\sigma=$ 0.006 and $\sigma=0.02$ )

\begin{tabular}{crrrr}
\hline Projections & \multicolumn{2}{c}{$\sigma=0.006$} & \multicolumn{2}{c}{$\sigma=0.02$} \\
\hline & DRTV & ADRTV & DRTV & ADRTV \\
15 & 40.66 & 42.01 & 36.78 & 36.57 \\
20 & 43.72 & 45.27 & 38.07 & 37.96 \\
30 & 48.03 & 49.38 & 40.48 & 40.02 \\
40 & 49.44 & 50.31 & 40.70 & 40.60 \\
60 & 51.30 & 51.61 & 40.74 & 40.73 \\
\hline
\end{tabular}


Indeed, for a relatively low with low-level additive noise $(\sigma=0.006)$, the PSNR values are enhanced in an amount of $1.31 \mathrm{~dB}$ for 60 projections to $1.35 \mathrm{~dB}$ for 15 projections. In this case, the quality of the reconstructed image is close to that of the original one. However, for a high noise level $(\sigma=0.02)$, and for several values of the projection number, the DRTV and ADRTV methods give similar PSNR values of the reconstructed image. Therefore, the minimization of the total variation associated to the positivity constraint (ADRTV algorithm) leads to significant enhancement of the reconstruction results in the case of a low number of projection data provided that the noise level remains reasonable. Otherwise, no noticeable enhancement can be expected.

b. Application to real images

The robustness of the proposed algorithm is testing by we apply them to image obtained by an X-ray generator. The examined sample is an electronic circuit. We present in Fig. 9, the reconstructed results of this image. The intensity profile of line 120 of the image which is 1D signal is presented in Fig. 10.

(a)

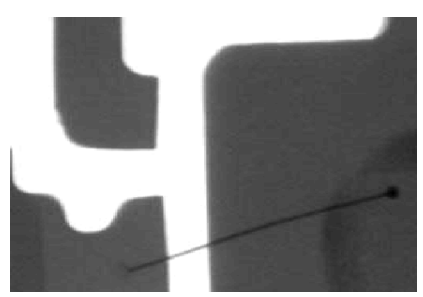

(b)

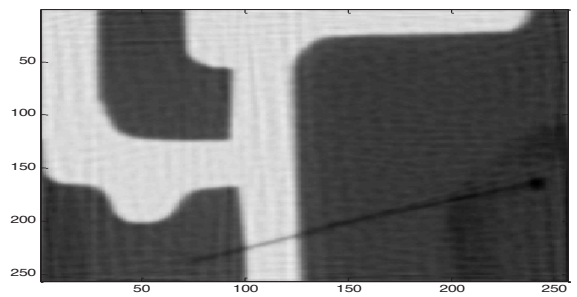

(c)

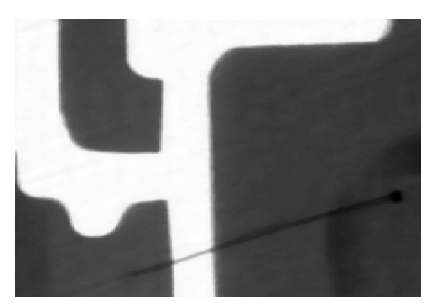

Fig. 9: Real image (a) and Obtained results using 30 projections. (b) Reconstructed image using DRTV (c) Reconstructed image using ADRTV.

(a)

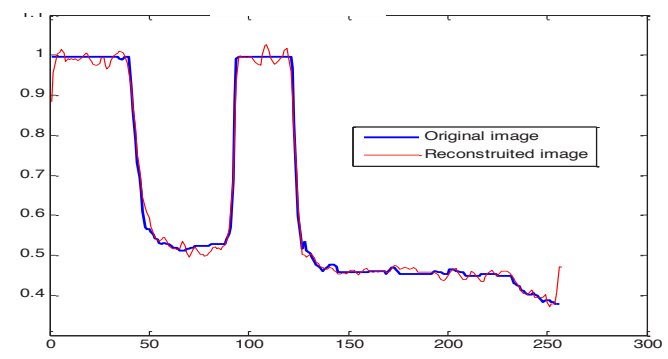

(b)

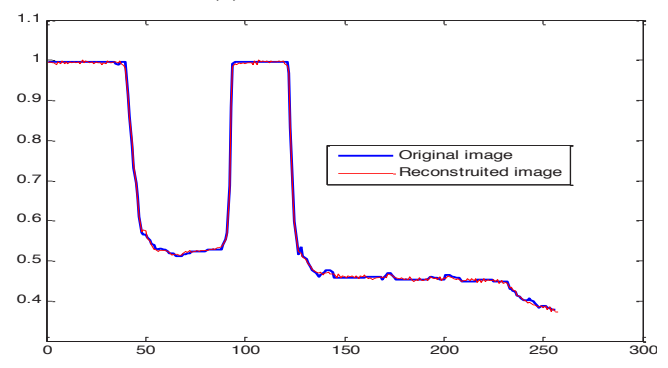

Fig. 10. Line intensity profile (a): Reconstructed image using DRTV (b): Reconstructed image using ADRTV.

As it can be seen from the reconstructed profiles, the ADRTV algorithm not only reduces the noise level but preserves the edge contours. The DR method can be improved by the use of total variation regularization, an adaptative parameter $\lambda$, and a positive constraint, provide reasonably good image quality

\section{Conclusion}

Our goal is to reconstruct an X-ray tomographic image in the case of a limited number of projections. We have proposed a new technique (ADRTV) in this condition. We have compared this technique with two other algorithms: DR and DRTV. We have made some simulations using synthetic images and real radiographic X-ray ones. The performance evaluation of the simulations used the PSNR and the relative error. We note that the DR method can be improved by using smoothing total variation regularization and an adaptative parameter $\lambda$ combined to a positive constraint, provide reasonably good image quality and more accurate estimation of the reconstructed image.

This concerned the visual reconstruction quality as well as the above mentioned parameters. In conclusion, the proposed ADRTV algorithm is able to reconstruct high-quality images though the limited number of projections in the x-rays tomography domain. 


\section{References}

[1] A.C. Kak, M. Slaney, Principles of computerized tomographic imaging ( IEEE Press, New York, 1988).

[2] Mark E. Davison. SIAM J. Appl. Math. 43(1983) 428-448.

[3] P. L. Combettes and J.C. Pesquet, Proximal Splitting Methods in Signal Processing, in: Fixed-Point Algorithms for Inverse Problems in Science and Engineering, ( New York: Springer-Verlag, 2010).

[4] J. Wang, T. Li and L. Xing, Med. Phys. 36 ( 2009) 252- 60.

[5] G. H. Chen, J. Tang and S. Leng, Med. Phys. 25 (2008) 660-663.

[6] A. Chambolle and T. Pock, JMIV, 40(2011), 120-145.

[7] P. L. Combettes, Journal of Convex Analysis. 16 (2009) 727-748.

[8] P.L. Combettes and V. Wajs. Multi. Model. And Simu. 4 (2005) 1168-1200.

[9] L. I. Rudin, S. Osher, E. Fatemi, Physica D. 60 (1992) 259-268.

[10] E. Candes, J. Romberg, T. Tao, IEEE Trans. Inf. Theory.52 (2006) 489-509.

[11] J. Douglas, H. H. Rachford. Trans. Amer. Math. Soc. 82(1956) 421-439.

[12] P. L.Combettes, J.-C.Pesquet, IEEE Journal of Selected Topics in Signal Processing. 1(2007) 564-574.

[13] C. R. Vogel, Computational Methods for Inverse Problems, (Society for Industrial and Applied Mathematics, Philadelphia, Pa, USA, 2002.

[14] Bardsley, J., Goldes,. Num. Alg. 57(2011), 255-271.

[15] Z. C. Yu, F. Noo, F. Dennerlein, A. Wunderlich, Lauritsch G and Hornegger. Phys. Med. Biol. 57 (2012).

[16] Jia X, Lou Y, Li R, Song W and Jiang S. Med Phys. 37,1757-1760 ( 2010).

[17] Yan M and Vese: Proceeding of SPIE Medical Imaging: Physics of Medical Imaging. 79612X (2011).

[18] Anthoine S, Aujol J F, Mélot C and Boursier Y. Inverse Problems and Imaging. 6,565-598 (2012).

[19] Sidky EY, Duchin Y and Pan XC, Med Phys. 38 (2011) 117-125

[20] Ritschl L, Bergner F, Fleischmann C and M. Kachelrie B, , Phys. Med Biol, 56 (2011) 1545-1561.

[21] L. Huang, L. Xiao and Z. Wei, Mathematical Problems in Engineering, 2011(2011).

[22] A. Chambolle and T. Pock, Journal of Mathematical Imaging and Vision. 40(2011) 120-145.

[23] A. Beck and M. Teboulle, IEEE TIP. 18 (2009) 2419-2434.

[24] K. Chen ·E. Loli Piccolomini ·F. Zama. NumerAlgor. 67 (2014) 73-92.

[25] Johnathan M. Bardsley John Goldes.. NumerAlgor.57(2011) 255-271.

T. Wang, K. Nakamoto, H. Zhang and H. Liu. IEEE Transactions on Nuclear Science. 64 (2017) 2742-2760. 